\title{
NOTAS SOBRE LA INTERSECCIONALIDAD ENTRE ETNICIDAD Y POLÍTICAS PENALES DE GÉNERO*
}

\section{Notes on the insersectionality of ethnicity and gendered criminal policies}

\author{
Dr. José Ángel Brandariz, Garcia ${ }^{* *}$ \\ "A la nueva cosecha de auto-proclamados \\ 'guardianes de los derechos de las mujeres', aquellos a los que nunca \\ hemos encontrado participando en, ni apoyando a, nuestros movimientos y \\ luchas a lo largo de los años, a éstos les decimos: ¡NO EN NUESTRO \\ NOMBRE! (...) Como feministas y mujeres realmente preocupadas por \\ la emancipación de las mujeres, no les permitiremos que hagan uso de "la \\ emancipación de las mujeres" para fines anti-migrantes, asimilacionistas, \\ islamofóbicos y etnocéntricos" \\ Nextgenderation, "Manifiesto NO EN NUESTRO NOMBRE"
}

Resumen: Las políticas penales de género, de especial difusión en el sistema punitivo español, presentan evidentes problemas en su relación interseccional con la etnicidad, como consecuencia de operar con una visión excesivamente unidimensional del sujeto "mujer" sobre el que se sustentan. Sin perjuicio de ello, tal vez dichas políticas podrían entenderse justificadas por sus efectos prácticos. El artículo indaga los riesgos de neorracismo que entraña esa deficiente relación interseccional, en particular la denominada teoría de los delitos culturamente motivados, y se interroga si puede justificarse desde una perspectiva utilitaria de la protección penal en clave de género.

Palabras claves: género - etnicidad - políticas penales - delitos culturalmente motivados.

Abstract: Gendered criminal policies, particularly prevalent in the Spanish criminal system, present clear problems in their intersectional relationship with ethnicity, due to an excessively unidimensional conception of the subject "woman", on which they are based. Nevertheless, perhaps these policies could be justified given their practical effects. The article explores the risks of neo-racism involved in this deficient intersectional relationship, namely, the so-called culturally-motivated crime theory. Additionally, the article asks whether such policies are justifiable from an utilitarian perspective regarding criminal protection in gendered grammar.

Keywords: gender - ethnicity - criminal policies - culturally-motivated crimes.

\footnotetext{
* El presente texto se ha realizado en el marco de los proyectos de investigación $\mathrm{n}^{\mathrm{o}}$ 10PXIB101082PR, subvencionado por la Consellería de Economía e Industria de la Xunta de Galicia, y n ${ }^{\circ}$ DER2011-24030JURI-, subvencionado por el Ministerio de Ciencia e Innovación, así como de la ayuda para grupos de investigación con potencial de crecimiento, otorgada por la Consellería de Cultura, Educación e Ordenación Universitaria de la Xunta de Galicia al grupo ECRIM de la Universidad de A Coruña.

** Profesor Titular de Derecho Penal, Universidad de A Coruña, España. Correo electrónico: branda@udc.es
}

Este artículo fue recibido el 8 de agosto de 2013, siendo aprobada su publicación con fecha 22 de agosto de 2013. 


\section{Introducción: España como sociedad crecientemente mestiza}

Un trabajo que pretenda hacer un análisis situado, es decir, centrado en un determinado tiempo y en un concreto lugar, sobre los retos que ha de afrontar el sistema penal en su relación con la etnicidad y el género, tal vez debe comenzar por una introducción sobre la creciente complejidad cultural de la sociedad española.

Aun sin desatender la innegable relevancia de la presencia tradicional de un significado segmento de población gitana, ${ }^{1}$ cabe reconocer que el sobresaliente incremento de la población extranjera residente, acontecido durante la primera década del siglo, permite considerar que la sociedad española es un conjunto humano crecientemente mestizo.

De acuerdo con los últimos datos del Instituto Nacional de Estadística (INE), ${ }^{2}$ a 1/I/2013 en España residían 5,520 millones de personas extranjeras, lo que equivalía al 11,7\% del total de la población residente. Desagregando esas cifras, tal como puede verse en la tabla 1, el mayor contingente de ese volumen de población extranjera está constituido por personas con nacionalidad de la UE (2,352 millones; $42,6 \%$ del total de extranjeros); junto a ellas, destacan también los números correspondientes a residentes con nacionalidad latinoamericana (1,412 millones; $25,6 \%$ total) y africana (1,096 millones; $19,9 \%$ total).

Tabla 1. Personas extranjeras residentes en España en 2013, por nacionalidades

\begin{tabular}{|l|l|l|}
\hline Nacionalidad & $\begin{array}{l}\text { Total personas residentes } \\
\text { (millones) }\end{array}$ & $\begin{array}{l}\text { \% sobre total extranjeros } \\
\text { residentes }\end{array}$ \\
\hline UE & 2,352 & $42,6 \%$ \\
\hline Europa no UE & 0,247 & $4,5 \%$ \\
\hline África & 1,096 & $19,9 \%$ \\
\hline Latinoamérica & 1,412 & $25,6 \%$ \\
\hline Asia & 0,376 & $6,8 \%$ \\
\hline Otros & 0,037 & $0,7 \%$ \\
\hline Total & 5,520 & $100 \%$ \\
\hline
\end{tabular}

De acuerdo con las estadísticas de Eurostat, estas cifras suponen que España era en 2011 el $5^{\circ}$ país de la UE en porcentaje de población extranjera residente, solo superado por Estados de pequeño tamaño o con situaciones políticas singulares. ${ }^{3}$

\footnotetext{
${ }^{1}$ Aun con todas las cautelas, LAPARRA (2011), pp. 27 y ss., estima que la cifra de población gitana en España se sitúa en torno a las 800.000 personas.

2 Vid. bttp:// wmw.ine.es/inebmenu/mnu_cifraspob.htm.

3 Vid. http://epp.eurostat.ec.europa.eu/portal/page/portal/population/data/main_tables. De acuerdo con las cifras mencionadas, España solo era superado en 2011 en este apartado por Estonia, Luxemburgo, Chipre y Letonia.
} 
Si la comparación se realiza con los otros Estados mayores de la UE, España presenta una proporción de población residente de nacionalidad extranjera claramente superior a la de Alemania $(8,8 \%)$, Italia $(7,5 \%)$, Reino Unido $(7,1 \%)$ o Francia $(5,8 \%){ }^{4}$

Como se ha señalado, este notable volumen de población extranjera residente en España es debido al importantísimo proceso migratorio experimentado en el país durante 2000-2011, en el que, como puede verse en la tabla 2, el número de foráneos censados en España pasó de 0,923 millones al inicio del periodo a 5,751 a su término. ${ }^{5}$ En el marco de la crisis, solo en 2011-2012 se ha experimentado una primera reducción, de cierta relevancia, del total de población extranjera residente.

Tabla 2. Evolución de la población extranjera residente en España (2000-2013)

\begin{tabular}{|l|l|l|}
\hline Año (1 enero) & $\begin{array}{l}\text { Población extranjera } \\
\text { (total/millones) }\end{array}$ & Población extranjera (\%/total) \\
\hline $\mathbf{2 0 0 0}$ & 0,923 & $2,2 \%$ \\
\hline $\mathbf{2 0 0 1}$ & 1,370 & $3,3 \%$ \\
\hline $\mathbf{2 0 0 2}$ & 1,977 & $4,7 \%$ \\
\hline $\mathbf{2 0 0 3}$ & 2,664 & $6,2 \%$ \\
\hline $\mathbf{2 0 0 4}$ & 3,034 & $7,0 \%$ \\
\hline $\mathbf{2 0 0 5}$ & 3,730 & $8,4 \%$ \\
\hline $\mathbf{2 0 0 6}$ & 4,144 & $9,2 \%$ \\
\hline $\mathbf{2 0 0 7}$ & 4,519 & $9,9 \%$ \\
\hline $\mathbf{2 0 0 8}$ & 5,268 & $11,4 \%$ \\
\hline $\mathbf{2 0 0 9}$ & 5,648 & $12,0 \%$ \\
\hline $\mathbf{2 0 1 0}$ & 5,747 & $12,2 \%$ \\
\hline $\mathbf{2 0 1 1}$ & 5,751 & $12,2 \%$ \\
\hline $\mathbf{2 0 1 2}$ & 5,711 & $12,1 \%$ \\
\hline $\mathbf{2 0 1 3}$ & 5,520 & $11,7 \%$ \\
\hline & & \multicolumn{1}{|l}{ Fuente: Elaboración propia a partir de datos del INE } \\
\hline
\end{tabular}

Todas estas cifras permiten sugerir que, en el marco de este fenómeno migratorio relativamente reciente, España se ha consolidado como una sociedad innegablemente plural, también en sentido cultural.

No obstante, para acabar de perfilar la imagen que sirve de sustrato al análisis situado que se pretende en el texto es necesario detenerse en la composición por sexo de esa población foránea. De acuerdo con las mismas

\footnotetext{
${ }^{4}$ Datos tomados de la misma fuente estadística de Eurostat. No puede desatenderse el hecho de que algunos de estos países mayores de la UE, como consecuencia de haber experimentado procesos migratorios de gran alcance temporal, presentan cifras elevadas de población residente originariamente extranjera. No obstante, también en este punto España se muestra por encima de dichos Estados; en 2010, el 14\% de la población residente en España había nacido en el extranjero, frente al 12,9\% de EE.UU., al 12\% de Alemania, al 11,3\% de Reino Unido, al 11,1\% de Francia o al 8\% de Italia (Fuente: datos citados de Eurostat, y UnitedStatesCensus Bureau [http:/ / mnnv.census.gov/population/foreign/]).

${ }^{5}$ Datos tomados de las cifras de población del INE (http://wmw.ine.es/inebmenu/mnu_cifraspob.htm).
} 
Brandariz - Notas sobre la interseccionalidad entre etnicidad y políticas penales de género

estadísticas poblacionales del INE, a 1/I/2013 la proporción de mujeres era superior entre la población de nacionalidad española $(51,1 \%)$ que entre los residentes con nacionalidad extranjera (48,5\%), como puede verse en la tabla 3. Ese mismo gráfico permite comprobar que las tasas de población femenina divergen de forma notable en función de la concreta nacionalidad foránea, con porcentajes muy significativos entre los latinoamericanos y los europeos no comunitarios, y llamativamente bajos entre los africanos y los asiáticos.

Tabla 3. Porcentajes de población femenina en 2013, por nacionalidades

\begin{tabular}{|l|l|}
\hline Nacionalidad & Porcentaje población femenina (\%/total) \\
\hline Total población & $50,8 \%$ \\
\hline Españoles & $51,1 \%$ \\
\hline Extranjeros (total) & $48,5 \%$ \\
\hline UE & $48,2 \%$ \\
\hline Latinoamericanos & $56,8 \%$ \\
\hline Africanos & $38,7 \%$ \\
\hline Europeos no UE & $57,1 \%$ \\
\hline Asiáticos & $41,3 \%$ \\
\hline
\end{tabular}

Fuente: Elaboración propia a partir de datos del INE

En suma, esta breve singladura estadística permite revisar dos hechos en absoluto desconocidos: que España cuenta con un importante volumen de población extranjera, y que dentro de ella la presencia de mujeres es relativamente semejante a la que se registra entre los habitantes de nacionalidad española. Ambas circunstancias prefiguran el interés de abordar el estudio de la relación entre diversidad cultural y género desde la perspectiva del sistema penal español.

\section{Etnicidad, género y sistema penal}

\section{II.1. Parámetros habituales del debate}

No parece aventurado entender que el debate sobre la relación entre sistema penal, diversidad y género ha tendido a centrarse, en gran medida en el examen de lo que se ha dado en llamar la delincuencia culturalmente motivada (en adelante, DCM). Este tópico de análisis viene a señalar que las sociedades occidentales experimentan cierto volumen de criminalidad protagonizada por personas pertenecientes a minorías, y que tal delincuencia estaría impulsada por ciertos parámetros culturales de esos grupos humanos. No resulta tampoco especialmente llamativo que ese modo de percibir una determinada delincuencia de las minorías recurra a las teorías criminológicas de las subculturas y de los conflictos culturales para explicar estos hechos. $^{6-7}$ Esta perspectiva considera que ejemplos paradigmáticos de esa DCM

${ }^{6}$ VÁZQUEZ GONZÁLEZ (2010), pp. 127 y ss. 
podrían ser la mutilación genital femenina, la poligamia, los homicidios por honor familiar o el matrimonio forzado. ${ }^{8}$

Esta breve introducción a la tesis permite constatar varios de sus elementos implícitos. En primer lugar, que en dicha teoría el paradigma del infractor no es del bomo oeconomicus, propio del Análisis Económico del Derecho, ${ }^{9}$ sino el del bomo islamicus. ${ }^{10}$ En efecto, resulta especialmente llamativo, en segundo lugar, que esta teoría solo parece prestar atención, de forma prioritaria o incluso única, a comportamientos lesivos que comúnmente se aparejan -con razón o sin ella- a la cultura islámica, desconsiderando otros hechos que puedan entenderse próximas a ciertas expresiones, v.gr., del cristianismo o del judaísmo. Junto a ello, y en tercer lugar, cabe constatar que el paradigma de la víctima es, en cambio, un sujeto femenino, en línea de principio perteneciente al mismo contexto cultural que el infractor.

De algún modo, es necesario admitir que este tipo de perspectiva goza en el contexto hispano de un eco más bien limitado. No ha tenido gran seguimiento en el ámbito académico, con alguna excepción significada, pero ciertamente mesurada en sus apreciaciones. ${ }^{11}$ La difusión social de este tipo de planteamientos es difícil de medir, pero ciertamente existe. Su penetración en el ámbito institucional tampoco parece muy acusada, sin perjuicio de posicionamientos ocasionales que destacan los males para la democracia de la cultura musulmana. ${ }^{12}$ Este limitado eco en España de los planteamientos de la tesis de la DCM no puede desconectarse de la pregunta referente a por qué la islamofobia no ha adquirido aquí los niveles observables en otras sociedades europeas. Sin perjuicio de la complejidad del interrogante, la respuesta ha de indagarse mediante el examen de las particularidades locales de los procesos sociales y culturales de selección de lo que, en inveterada expresión criminológica, se ha denominado como demonios populares. ${ }^{13}$

A pesar de estas limitaciones en su difusión, las huellas de la tesis de la DCM pueden verse, de uno u otro modo, en la legislación penal vigente. Sin duda dicho rastro es evidente en la tipificación específica del delito de mutilación genital

\footnotetext{
7 Vid., extensamente sobre ello, MONCLús MASÓ (2008), pp. 137 y ss.

${ }^{8}$ BRION (2010), p. 80.

${ }^{9}$ De HaAn y Vos (2003), pp. 31 y ss.

${ }^{10}$ BRION (2010), p.88.

11 VÁZQUEZ GONZÁLEZ (2010) hasta donde se alcanza a ver, el trabajo citado es el que de forma más detenida $-\mathrm{y}$, por cierto, rica y compleja- desarrolla la perspectiva teórica mencionada. Otros estudios, como CORNACCHIA y SÁNCHEZ-OSTIZ (2012) o CARNEVALI (2007), distan de abordarlo con la misma densidad.

12 Vid., a modo de referencia, las declaraciones del entonces diputado del PP Ignacio Cosidó, recogidas en el diario Público de 1/VII/2008.

13 Una evidencia de las particularidades hispanas en relación con la selección de enemigos apropiados podría ser la sorprendente pervivencia del debate sobre la autoría de los atentados del 11/III/2004 en Madrid (vid., sobre ello, OBSERVATORIO METROPOLITANO (2012), pp. 65 y ss.).
} 
(art. 149.2 CP). ${ }^{14}$ Si cupiese alguna duda de ello, cabe reparar en que dicho tipo se introdujo en el cuerpo legal mediante la L.O. 11/2003, de 29/IX, que no en vano se denominó "de medidas concretas en materia de seguridad ciudadana, violencia doméstica e integración social de los extranjeros". La Exposición de Motivos de dicha ley no puede ser más explícita en relación con la imaginería que subyace a la modificación normativa, cuando señala que "la reforma se plantea desde el reconocimiento de que con la integración social de los extranjeros en España aparecen nuevas realidades a las que el ordenamiento debe dar adecuada respuesta. Asi, como novedad igualmente reseñable, se tipifica el delito de mutilación genital o ablación. Y ello porque la mutilación genital de mujeres y niñas es una práctica que debe combatirse con la máxima firmeza, sin que pueda en absoluto justificarse por razones pretendidamente religiosas o culturales".

No obstante, no es este el único extremo de la legislación penal española en el que puede vislumbrarse la influencia de la tesis de la DCM. En el momento de redactar estas páginas el Ministerio de Justicia ha presentado un amplio Anteproyecto de reforma del CP. En dicho texto se contempla la tipificación específica, como modalidad cualificada de coacciones, del matrimonio forzado (nuevo art. 172bis CP). ${ }^{15}$ Junto a ello, no cabe dejar de mencionar la figura vigente de trata de seres humanos (art. 177 bis CP), con fines -entre otros- de explotación sexual, en la cual, junto a los compromisos internacionales, subyace la semántica de la prostitución coactiva de mujeres extranjeras. ${ }^{16}$

Más allá de la influencia en la legislación punitiva española de este modo habitual de analizar la relación entre sistema penal, diversidad cultural y género, una característica de la máxima relevancia de esta orientación analítica es la acusada influencia que en ella ejerce un determinado tipo de feminismo. En efecto, si bien no es fácil percibir cuál es el sustrato político-cultural más relevante de este tipo de perspectiva, parece evidente que en él influye sobremanera un cierto feminismo, que, en aras de reconocer la innegable pluralidad de este movimiento, y a falta de una mejor expresión, podría denominarse con el anglicismo mainstream.

Sin voluntad alguna de incurrir en reduccionismos, los planteamientos de esta orientación feminista al debate analizado podrían sintetizarse en algunas ideas. ${ }^{17}$ En primer lugar, el feminismo mainstream considera que los condicionamientos sociales, culturales y religiosos de las mujeres migrantes, o pertenecientes a minorías no occidentales, esconden situaciones de discriminación.

\footnotetext{
14 VÁZQUEZ GONZÁLEZ (2010), pp. 163 y ss.

${ }^{15}$ No obstante, la Exposición de Motivos del Anteproyecto no recurre en su justificación de la reforma a la retórica de la DCM, sino a los compromisos internacionales (citando, al efecto, la Directiva 2011/36/UE, del Parlamento Europeo y del Consejo, de 5/IV/2011, relativa a la prevención y lucha contra la trata de seres humanos y a la protección de las víctimas, así como la Convención de la ONU sobre la eliminación de todas las formas de discriminación contra la mujer, de 18/XII/1979), una cita que remite al lenguaje de la violencia de género.

${ }^{16}$ IgLESIAS SkUlj (2012), 61 y ss.

17 A continuación se sigue fundamentalmente el completo análisis realizado por VÁZQUEZ GONZÁLEZ (2010), pp. 94 y ss.
} 
En este sentido, tales condicionantes potencian la victimización de estos grupos de mujeres por razón de género.

En segundo lugar, y en relación con ello, este planteamiento considera que en el análisis de las cuestiones en juego, es decir, la diversidad cultural, las migraciones y la delincuencia vinculada de uno u otro modo a ellas, es necesario adoptar una perspectiva de género. El feminismo mainstream destaca, con razón, que en el análisis de fenómenos como las migraciones o la victimización delictiva se asume el punto de vista de un sujeto pretendidamente neutro, que en realidad es masculino (y ciudadano). En consecuencia, se hace necesario enfocar tales hechos sociales desde la óptica de las mujeres que los experimentan, los protagonizan o los padecen. La adopción de esa perspectiva permitiría, por lo demás, comprobar que las mujeres pertenecientes a las minorías sufren un doble, o triple, proceso de discriminación: como mujeres, como mujeres migrantes y, en su caso, como mujeres migrantes irregulares. A ello se añade que en ese complejo proceso de subordinación, la discriminación de género se ve reforzada en el contexto de determinadas culturas.

Más allá de la afirmación de todo este planteamiento, el feminismo mainstream se posiciona frente a otras formas posibles de entender la relación entre género, diversidad cultural y delincuencia. En efecto, se entiende que la mujer (minoritaria y/o migrante) es la gran perdedora de cualquier tipo de relativismo cultural. No en vano, se considera que la defensa acrítica de la valía de determinadas culturas supone, consciente o inconscientemente, mantener graves pautas de discriminación de género que afectan a las mujeres pertenecientes a las minorías. Por si cupiese alguna duda sobre la forma de afrontar esas discriminaciones, esta orientación feminista considera que en la medida en que suponen graves riesgos para la integridad y la dignidad de las mujeres es necesario luchar contra ellas -también-con los instrumentos punitivos.

Este recurso al sistema penal se ve reforzado por la consideración tal vez de mayor densidad simbólica de estos planteamientos del feminismo mainstream: las discriminaciones de las mujeres derivadas de tradiciones culturales son manifestaciones de violencia de género. En tanto que tales, y esta es seguramente la conclusión más problemática de todo el análisis, las mujeres que pertenecen a esas tradiciones culturales no tienen verdadera capacidad de decidir en relación con su inserción en las prácticas discriminatorias, sino que son víctimas de esas expresiones de violencia de género.

\section{II.2. Insuficiencias de los parámetros habituales de debate}

A pesar de la difusión de los planteamientos de la DCM y, sobre todo, de la teoría feminista que fundamenta y acompaña ese planteamiento, los parámetros de debate que se han sintetizado presentan significativas insuficiencias. De hecho, la trascendencia y solidez de esas insuficiencias es tal que sorprende que 
la crítica de los parámetros habituales de discusión no haya alcanzado un eco similar al de esos planteamientos.

La primera de las insuficiencias, de consideración obligada en la medida en que se está abordando una cuestión político-criminal, es de orden utilitario o -si se quiere- práctico. Se trata de la desatención de esa orientación de pensamiento por la verdadera eficacia de los planteamientos criminalizadores de determinadas formas de DCM. Frente a esa desconsideración, un análisis que persiga traducir dicha tesis en la adopción de políticas públicas debe necesariamente interrogarse por la entidad del problema. En esta línea, una pregunta que no se intuye en absoluto inoportuna, sino imprescindible, sería ¿Cuántos hechos de mutilación genital femenina, de matrimonios forzados, de poligamia, etc., culturalmente motivados, se dan en el contexto español?

Más allá de una posible contestación, tan reduccionista como evidente, en la línea de enunciar que es imposible saberlo, el problema de esta pregunta es que los indicios que permiten responderla no parecen estar en consonancia con la entidad del debate. En efecto, hasta donde se alcanza a ver, la primera condena en España por mutilación genital femenina, que terminó en una sentencia en gran medida singular, es de noviembre de $2011 .^{18}$ No es necesario enfatizar que se trata de una resolución emitida más de ocho años después de la entrada en vigor del actual art. 149.2 CP. Parece, en cambio, necesario llamar la atención sobre el hecho de que esa disonancia entre la preocupación pública y los resultados de persecución penal se da en relación con un tipo específico de lesiones que presenta al menos dos circunstancias diferenciales que deberían facilitar, de manera muy notable, su punición efectiva. En primer lugar, el tipo del art. 149.2 CP, cuando tenga como víctimas mujeres, es una de las poquísimas figuras que -si bien con particularidades- pueden ser perseguidas extraterritorialmente, de acuerdo con la regulación del principio de justicia universal del art. 23.4 LOPJ. En segundo lugar, ese género de lesiones graves constituye también uno de los pocos fenómenos delictivos que cuenta con cuerpos policiales especializados en su investigación y persecución. ${ }^{19}$ Pues bien, a pesar de la disposición de estos recursos normativos, financieros y humanos, los resultados en términos punitivos son muy llamativos en un sistema penal que produce más de 250.000 condenas anuales.

Precisamente, si se acude a las estadísticas de condenados del INE ${ }^{20}$ las insuficiencias de orden utilitario de la tesis de la DCM se acrecientan. En efecto, en un sistema penal español que condenó en 2011 la comisión de casi 274.000 delitos, los ilícitos de trata penados fueron cinco (esto es, el 0,0018\% de los injustos objeto de condena), y los de matrimonios ilegales siete (el $0,0025 \%$ del

\footnotetext{
18 PÉReZ Del VAlLE (2012), p. 95 y s.

${ }^{19}$ Es bien conocido el trabajo en este ámbito de los Mossosd'Esquadra, que anuncian periódicamente en la prensa resultados de su gestión que no se corresponden en absoluto con las condenas efectivas. Sobre el particular, vid. especialmente http:// wmw20.gencat.cat/docs/Adjucat/Documents/ARXIUS/protocolo_mut.pdf.

${ }^{20} \mathrm{Vid}$. http:// www.ine.es/inebmenu/mnu_justicia.btm.
} 
total). A mayor abundamiento, tampoco parece que los datos disponibles permitan corroborar de forma pacífica la perspectiva de género en la materia, ya que las cifras del INE informan de que en 2011 fueron condenadas dos mujeres por trata y otras dos por matrimonios ilegales.

En relación con las conclusiones que cabe derivar de estas cifras es necesario hacer una clarificación. Con ellas no se pretende insinuar que cada uno de esos hechos objeto de condena no sea preocupante, ante todo -obviamentedesde la perspectiva de sus víctimas. No obstante, desde una perspectiva de interés público no es menos evidente que los debates político-criminales que no se sustentan mínimamente en análisis empíricos y -cabría añadir- de utilidad y eficacia presentan graves insuficiencias, por mucho que ese sea el modo inveterado de evolución del sistema penal español. Dicho de otro modo, es necesario denunciar graves hechos lesivos cometidos contra mujeres, pero también lo es criticar que se reiteren planteamientos sin detenerse a indagar cuál es su verdadero impacto en la realidad del funcionamiento del sistema punitivo y, más allá, de la evolución de la propia sociedad.

De hecho, la mencionada insuficiencia, que por su carácter utilitario podría pensarse menor, permite formular una hipótesis más preocupante, que prefigura las demás disfunciones de la tesis criticada. Esa hipótesis consiste en intuir que la Política criminal sustentada en la teoría de la DCM es de escasa utilidad para la prevención de delitos, pero es notablemente útil para construir dinámicas de alteridad y de discriminación por razones de etnia, de origen o, si se quiere, culturales. ${ }^{21}$

Para llegar a desarrollar las bases de esta hipótesis, parece procedente detenerse previamente en algunos problemas epistemológicos que presenta la tesis analizada, y que en gran medida sientan las bases para que produzca esos lamentables efectos. Tales problemas epistemológicos se derivan de dos reduccionismos que subyacen a la teoría de la DCM.

El primero de esos reduccionismos tiene que ver con el concepto de cultura asumido por la tesis. Tal vez de forma inconsciente, la teoría criticada parte de una noción tendencialmente estática e inmutable de cultura, frente a un concepto vivo, abierto y en mutación, mucho más consonante con la realidad de los procesos culturales. En este sentido, el planteamiento de la DCM se construye sobre una insostenible reificación de la cultura, en un ejercicio de lo que bien podría denominarse fundamentalismo cultural. ${ }^{22}$

\footnotetext{
${ }^{21}$ BRION (2010), pp. 83 y ss.

22 STOLCKE (1995), pp. 4 y ss.
} 
Ese carácter fundamentalista no es lo único que aproxima la tesis analizada a una operación narrativa como la realizada por Huntington. ${ }^{23}$ De hecho, esa proximidad permite intuir las razones que hacen que la tesis de la DCM tenga tan buena acogida en entornos neoconservadores. Este planteamiento comparte con la teoría huntingtoniana, siquiera de forma inconsciente, la necesidad de reconstruir en el tiempo presente la figura de la alteridad, o del enemigo. En ese punto se muestra peligrosamente próxima a la asunción del axioma schmittiano ${ }^{24}$ según el cual la relación amigo-enemigo es un elemento fundamental del funcionamiento de un sistema sociopolítico. Tanto Huntington como la teoría de la DCM parecen asumir que en ese proceso de construcción de la alteridad el (varón) musulmán se presenta hoy como el enemigo idóneo por antonomasia.

No obstante, como se ha sugerido, el problema de este planteamiento, más allá de sus preocupantes efectos políticos, es que carece de sustento epistemológico. En efecto, las tesis que se basan en una noción estática, no viva, de cultura, y que derivan de ella conflictos y enemigos culturales, requieren al menos dos presupuestos. En primer lugar, la premisa de que cada subcultura integrante de una sociedad compleja se caracteriza por una homogeneidad normativa y cultural interna. En segundo lugar, los planteamientos objeto de crítica también requieren el presupuesto de la rivalidad entre esas subculturas homogéneas, es decir, necesitan asumir que la convivencia entre diferentes grupos culturales en una misma sociedad es, inevitablemente, muy difícil o imposible.

Siendo así, el problema epistemológico fundamental de estos planteamientos es que, como enseña todo el trabajo de la interculturalidad, no se corresponden con la realidad. Por una parte, si las sociedades del presente son complejas, junto a ello -más bien, por ello- son también sumamente fluidas $\mathrm{y}$, desde luego, híbridas. Por otra parte, la homogeneidad de cada cultura es irreal; las culturas son realidades vivas y en mutación, lo que explica que sean inherentemente plurales. Por lo demás, de forma expresa en Huntington e implícita en la tesis de la DCM, se produce una sobredeterminación de la influencia de la religión en la conformación de cada cultura, algo que carece de sentido tanto como las premisas antecedentes.

En consecuencia, este conjunto de aporías permite intuir que estamos, una vez más, ante un proceso de construcción del saber mediado por el poder; dicho con más claridad, la reificación cultural es insostenible en el plano del conocimiento, pero constituye una operación de gobierno especialmente cómoda, orientada a la gestión de la complejidad en sociedades de hibridación creciente.

\footnotetext{
${ }^{23}$ La tesis del choque de civilizaciones, popularizada por S. Huntington, aparece recogida en su famoso libro The Clash of Civilizations and the Remaking of World Order, Simon\&Schuster, New York, 1996, si bien existe una versión resumida anterior. Para una aproximación crítica a esta tesis, preñada de un esencialismo simplista, vid., por todos, YOUNG (2003), pp. 167 y ss. y pp. 187 y ss.; ŽIŽEK (2005), pp. 37 y ss.

${ }^{24}$ Sснмітт (2009), pp. 71 y ss. y 129 y ss.
} 
Sin perjuicio de todo ello, el uso de un concepto de cultura inapropiado no es el único problema epistemológico que afecta a la tesis de la DCM. La teoría se sustenta sobre un segundo reduccionismo, en este caso el relativo al sujeto del análisis feminista; esta concreta disfunción genera, además, evidentes problemas políticos.

En efecto, las consecuencias políticas del desafortunado empleo del concepto de mujer se intuyen ya cuando se repara en la singular relación entre culturas políticas que se manifiesta en el ámbito de la teoría estudiada. En ella, el feminismo mainstream tiene como extraña compañera de viaje a la islamofobia de corte neocon. Para comprobar esa conexión solo hace falta reparar en el eco que han recibido los planteamientos de la DCM en ciertos países europeos, señaladamente por su enunciación reiterativa por parte de personajes políticos como Pia Kjærsgaard, Pim Fortuyn, Geert Wilders o Ayaan Hirsi Ali, todos ellos destacados representantes del pensamiento neocon europeo de la última década. ${ }^{25}$

En esos responsables políticos se manifiesta esa particular interconexión entre xenofobia, islamofobia, neoconservadurismo y retórica feminista mainstream. Obviamente, no se trata de responsabilizar a ese vector del pensamiento de género de esta extraña amalgama. Con todo, no parece osado intuir que hay algunos elementos en la retórica del feminismo mainstream sobre la DCM que facilitan el encuentro con esas otras corrientes de pensamiento, y que constituyen límites no solo epistemológicos, sino también políticos, de su forma de abordar la materia.

El problema fundamental reside en una nueva reificación conceptual, que acompaña a la relativa a la cultura. En este caso, se trata del concepto de mujer. Es conocido que la preocupación de este sector del feminismo por construir un sujeto mujer unitario, y en singular, paga un elevado precio: el de desatender la pluralidad inherente a las mujeres, lo que, dicho sea de paso, supone desatender la realidad.

En consonancia con ello, el feminismo mainstream incurre en su análisis de la DCM en un par de reduccionismos en cadena. Por una parte, tiende a reducir la imagen de los sujetos femeninos migrantes o pertenecientes a minorías a la correspondiente a la mujer árabe. En segundo lugar, reduce a la mujer árabe a la imagen de la mujer sometida por su cultura. Como consecuencia, en su esfuerzo por mantener una visión universalista de la mujer, y del discurso feminista, el pensamiento de género mainstream se arriesga a asumir, inconscientemente, una visión neocolonial de una amplia pluralidad de mujeres.

Junto a ello, la perspectiva del feminismo mainstream sobre la DCM paga el precio de desatender un conjunto de dinámicas de extraordinario interés, que deberían ser objeto no solo de su atención, sino también de su decidido apoyo. En primer lugar, la mencionada óptica unidimensional desconsidera los procesos de

${ }^{25}$ FEKETE (2009), pp. 77 y ss. 
transformación e hibridación cultural emprendidos por las mujeres minoritarias en contextos migratorios. En segundo lugar, y en relación con ello, el feminismo mainstream desatiende la agencia de las mujeres migrantes, que en su retórica se ven reducidas al impotente papel de víctimas. El efecto de ello es, obviamente, la despolitización de las mujeres pertenecientes a las minorías, una circunstancia que crea el riesgo de que el feminismo mainstream se convierta en una suerte de pensamiento paternalista. ${ }^{26}$ De manera más específica, y en tercer lugar, el mencionado sector de pensamiento desconoce en su retórica los procesos de empoderamiento y liberación de género propios del feminismo musulmán y, más general, de los feminismos del tercer mundo.

Parece evidente que todo ello es insostenible desde una perspectiva epistemológica. De hecho, esa insostenibilidad se muestra de manera más descarnada cuando, conociendo la teoría de género, se repara en las sólidas aportaciones realizadas en las tres últimas décadas por el feminismo negro, el postestructuralista o el postcolonial. ${ }^{27}$ No obstante, esta operación reduccionista es también muy cuestionable desde una perspectiva política, en la medida en que genera el efecto de alimentar las diferencias y las desconfianzas por parte de los feminismos menos institucionalizados.

Estas preocupantes consecuencias del pensamiento feminista más convencional sobre la materia estudiada se complementan con el principal problema político que produce la tesis de la DCM. Como se avanzó previamente, la Política criminal sustentada en tales planteamientos es de escasa utilidad para la prevención delictiva, pero sumamente eficaz para construir categorías de alteridad y estigmatizar a las minorías migrantes. En efecto, parece evidente que la teoría de la DCM estigmatiza a los grupos minoritarios, y, por cierto, no solo a los varones pertenecientes a ellos. De esta forma, tiende a presentar los procesos de heteroexclusión que les afectan como si fuesen dinámicas de autoexclusión de la sociedad de acogida; dicho de otro modo, contribuye a justificar discriminaciones ilegítimas como si fuesen distinciones legítimas.

En línea de principio, se supone que toda construcción de categorías de alteridad resulta útil para el mantenimiento de la cohesión social, en tanto que conformación de un exterior constitutivo del grupo. ${ }^{28}$ No obstante, habría que admitir que esa cohesión es incompleta o, si se quiere, demediada, en la medida en que margina a los grupos estigmatizados. ${ }^{29}$ De hecho, se trataría de una suerte de cohesión en negativo, que opera mediante dicotomías. Las más evidentes de ellas serían las oposiciones entre autóctonos y migrantes o, más en concreto, entre musulmanes y occidentales.

\footnotetext{
${ }^{26}$ Op. cit., pp. 96 y ss.

27 Sobre estas orientaciones, vid., a modo de referencia, HOOKS et al. (2004); JABARDO (2012); MOHANTY (2008), pp. 69 y ss.

${ }^{28}$ BAUMAN (2010), p. 39 y s.; Young (2007), p. 141 y s.

29 TSOUKALA (2002), 76 y s.
} 
Las construcciones semióticas que se derivan de esas oposiciones son claramente disfuncionales. Del mismo modo que se atribuye a los musulmanes y a su cultura una condición liberticida y, en último término no civilizatoria, se refuerza el simulacro de atribución del respeto a los derechos humanos por parte del grupo mayoritario autóctono. ${ }^{30}$ La narrativa que, en último término, se deriva de ello no puede ser más patriarcal y neocolonial: el uso del Derecho penal para combatir la DCM aparece como una historia de varones blancos/mayoritarios salvando a mujeres no blancas/minoritarias de los riesgos que suponen los varones no blancos. A mayor abundamiento, otro efecto disfuncional de todo ello es que la retórica de la DCM contribuye a desplazar la atención de las graves violaciones de derechos humanos cometidas por motivos de género por personas autóctonas.

En suma, la teoría de la DCM no solo se sustenta en bases epistemológicamente débiles y contribuye a socavar la potencia de los feminismos, sino que refuerza política y socialmente la xenofobia y la islamofobia. Además, como toda tesis de efectos xenófobos sustentada en nociones estáticas de cultura, contribuye, aún a su pesar, a la difusión de la forma hoy prevalente de racismo: el racismo cultural o neorracismo. ${ }^{31}$

Por si todo ello no fuese suficiente, desde la perspectiva más específica de la Política criminal, la tesis de la DCM conduce a una evidente distorsión en el análisis de la vinculación entre diversidad cultural, género y sistema penal, a saber, aquella que se deriva de perder de vista que la relación de las minorías con el campo punitivo está mediada, de forma inherente por condiciones discriminatorias ajenas a la cultura, y sustentadas ante todo en la desigualdad de los marcos normativos de derechos. A este último problema político(-criminal) de la tesis de la DCM se dedica el siguiente epígrafe, a modo de conclusión.

\section{Conclusión: Hacia una reconsideración de las relaciones entre etnicidad, género y sistema penal}

Retomando la última idea enunciada, el discurso de la DCM, con su difusión, dificulta la conformación de una perspectiva menos unidimensional de las relaciones entre diversidad cultural, género y sistema penal. A los efectos de comenzar a pensar un camino de análisis para esa reconfiguración de la materia, cabría partir de dos premisas. En primer lugar, es necesario admitir que las particularidades de la criminalidad de las minorías o de los migrantes tienen menos que ver con las características de tales personas que con la influencia que ejerce un estatuto normativo claramente subordinado sobre sus procesos de subjetivación. ${ }^{32}$ En segundo lugar, si se asume este presupuesto cabe admitir que es tanto o más relevante la relación de la

\footnotetext{
${ }^{30}$ BRION (2010), pp. 86 y ss.

${ }^{31}$ BALIBAR y WALLERSTEIN (1991), pp. 31 y ss.; BRAH (2011), p. 186 y pp. 197 y ss.

32 PALIDDA (2008), p. 118 y s.
} 
Brandariz - Notas sobre la interseccionalidad entre etnicidad y políticas penales de género

diversidad cultural y el género con la respuesta punitiva a la delincuencia que la vinculación de aquellas variables con la propia criminalidad.

El sentido de este planteamiento puede percibirse prestando atención a los datos seleccionados en las tablas 4 y 5 .

Tabla 4: Distribución de delitos objeto de condena en 2011, por nacionalidad

\begin{tabular}{|l|l|l|l|l|l|l|}
\hline & Españoles & UE & $\begin{array}{l}\text { Europeos no } \\
\text { UE }\end{array}$ & Africanos & Americanos & Asiáticos \\
\hline Total delitos & $73 \%$ & $8,2 \%$ & $0,9 \%$ & $7,1 \%$ & $10 \%$ & $0,8 \%$ \\
\hline Agresiones sexuales & $53,7 \%$ & $9,9 \%$ & $1 \%$ & $\mathbf{1 5 , 4 \%}$ & $\mathbf{1 9 , 1 \%}$ & $1 \%$ \\
\hline Hurtos & $62,6 \%$ & $\mathbf{2 0 , 8} \%$ & $\mathbf{1 , 9} \%$ & $6,8 \%$ & $6,7 \%$ & $0,8 \%$ \\
\hline Tráfico de drogas & $53,3 \%$ & $7,3 \%$ & $0,4 \%$ & $\mathbf{2 4 , 2} \%$ & $13,1 \%$ & $\mathbf{1 , 6} \%$ \\
\hline $\begin{array}{l}\text { Falsedades } \\
\text { documentales }\end{array}$ & $49,1 \%$ & $10,1 \%$ & $\mathbf{2 , 4} \%$ & $\mathbf{2 4 \%}$ & $9,8 \%$ & $\mathbf{3 , 7} \%$ \\
\hline
\end{tabular}

Fuente: Elaboración propia a partir de datos del INE

La primera de ellas, elaborada a partir de los datos de la estadística de condenados 2011 del INE, ${ }^{33}$ hace una comparación entre las tasas generales de condena en 2011 por grandes grupos de nacionalidades y las correspondientes a ciertas figuras delictivas, en las que se aprecia una acusada sobrerrepresentación de los condenados extranjeros. En particular, llama la atención la categoría de las falsedades documentales, en la que, con un número de condenas no desdeñable ${ }^{34}$, los europeos extracomunitarios multiplican por 2,7 su tasa de sanción general, los africanos por 3,4, y los asiáticos por 4,6.

Tabla 5: Distribución de penas de prisión por sexo y nacionalidad, 2011

\begin{tabular}{|l|l|l|l|l|l|l|l|}
\hline & $\begin{array}{l}\text { Total } \\
\text { mujeres }\end{array}$ & Españolas & UE & $\begin{array}{l}\text { Europeas } \\
\text { no UE }\end{array}$ & Americanas & Africanas & Asiáticas \\
\hline Total penas & $\mathbf{9 , 1} \%$ & & & & & & \\
\hline $\begin{array}{l}\text { Penas prisión } \\
\text { (total) }\end{array}$ & $\mathbf{1 0 , 5 \%}$ & $7,7 \%$ & $1,1 \%$ & $0,2 \%$ & $1,2 \%$ & $0,5 \%$ & $0,1 \%$ \\
\hline $\begin{array}{l}\text { Penas prisión } \\
\mathbf{0 - 2} \text { años }\end{array}$ & $10,6 \%$ & $7,7 \%$ & $1,1 \%$ & $0,2 \%$ & $1,1 \%$ & $0,5 \%$ & $0,1 \%$ \\
\hline $\begin{array}{l}\text { Penas prisión } \\
\text { más de 5 años }\end{array}$ & $\mathbf{1 1 , 3 \%}$ & $4,2 \%$ & $1,6 \%$ & $0,1 \%$ & $\mathbf{5 , 4} \%$ & $0,1 \%$ & $0 \%$ \\
\hline
\end{tabular}

Fuente: Elaboración propia a partir de datos del INE

La tabla 5, partiendo de la misma fuente estadística, ofrece una panorámica sobre la distribución de las penas de prisión, como sanciones más graves de nuestro ordenamiento, en relación con las variables de sexo y nacionalidad, y en comparación

\footnotetext{
33 Vid. http:// www.ine.es/inebmenu/ mnu_justicia.btm.

34 De acuerdo con los datos de la mencionada estadística del INE, las condenas en 2011 por falsedades documentales fueron 5.795, lo que representa el 3,7\% de total de delitos objeto de condena con exclusión de los ilícitos contra la seguridad vial.
} 
con la tasa general de punición de las mujeres. En ella se evidencia que el porcentaje de mujeres penadas se incrementa a medida que asciende la escala de severidad de las sanciones, alcanzando la mayor cota en relación con las condenas de prisión de larga duración. Junto a ello, la tabla ofrece un dato aún más significativo: si las mujeres de nacionalidad americana constituyeron el 1,2\% de las personas condenadas a prisión en 2011 -es decir, aproximadamente 1 de cada 80-, su porcentaje ascendió a un 5,4\% -esto es, 1 de cada 18- en el caso de las penas de prisión graves.

Estos datos ponen de relieve cuál es el campo de análisis de la relación entre diversidad cultural, género y sistema penal que se sugiere. La tarea que está por hacer consiste fundamentalmente en examinar de qué forma responde el sistema penal español ante el comportamiento no de sujetos anónimos sin atributos, sino de personas caracterizadas por concretos rasgos de género y de nacionalidad u origen. $\mathrm{Si}$ resulta evidente que las estadísticas de un sistema punitivo no reflejan las características de la criminalidad de un país, sino únicamente los resultados del funcionamiento de dicho sistema, ${ }^{35}$ hay buenas razones para indagar si el español es realmente neutro en relación con el género y el origen nacional. Las evidencias de la particular severidad con la que se trata a las mujeres, y a los hombres y mujeres de determinadas nacionalidades, en relación con ciertas penas y concretas figuras delictivas, son el mejor motivo para ello.

Esa tarea tiene un objetivo político innegable, y al menos tan digno como el pretendido por la teoría de la DCM: contribuir desde el ámbito académico a evitar que el sistema penal reproduzca y amplifique las discriminaciones, de género y de nacionalidad, presentes en el plano social. ${ }^{36}$

En tal labor el investigador habrá necesariamente de dar respuesta a dos retos mayores del modelo punitivo español vigente, que suponen severas discriminaciones por razón de nacionalidad, y que, si bien no incorporan directamente la variable de género, afectan también a las mujeres de origen no autóctono.

El primero de esos retos tiene que ver con reformular la actividad policial, en la línea de evitar que siga operando a partir de criterios de nacionalidad, étnicos o fenotípicos. Se trata de una cuestión capital para evitar la pervivencia de este tipo de discriminaciones en el sistema penal español, aunque solo sea porque la policía es la puerta de entrada por antonomasia al campo punitivo. ${ }^{37}$ La complejidad de la sociedad española del presente impide que se mantenga de forma acrítica una forma de funcionamiento policial propia de una época en la que se presumía que no existían diferencias étnicas o de color de piel entre los habitantes del Estado. El ámbito de análisis de esta materia tiene que ver, de forma evidente, con la constancia de la intensificación, al menos desde 2008, de redadas constantes contra migrantes en las principales ciudades españolas, así como con normas tan preocupantes como la orden

\footnotetext{
35 MONClús MASÓ (2008), p. 225 y s. y p. 228.

36 Op. cit., p. 229 y s.

37 GoOdEY (2006), p. 207.
} 
de la Jefatura policial de Madrid de cupos de detención (noviembre 2008) o la preocupante Circular $1 / 2010$ de la Comisaría General de Extranjería, hoy afortunadamente derogada por la 10/2012.38 Más allá de ello, este análisis de las prácticas policiales tiene que ver con la necesidad de considerar, en línea con el Dictamen del Comité de Derechos Humanos de la ONU de 27/VII/2009 -que condenó al Estado español-, y a diferencia de la STC 13/2001, de 29/I, que los rasgos somáticos o fenotípicos no pueden en ningún caso ser base admisible para justificar una determinada intervención policial. ${ }^{39}$ Por lo demás, esta tarea de estudio debería tener en cuenta la posibilidad de incorporar orientaciones antidiscriminatorias en la actuación policial semejantes a las que contempla el art. 95 de la Criminal Justice Act 1991 británica. ${ }^{40}$

El segundo reto fundamental de análisis de la relación entre la diversidad cultural y el sistema penal español se deriva de la necesidad de percibir que la pobre e inveterada imagen de la sociedad española como un conjunto humano étnica y culturalmente homogéneo contribuye a producir severas insuficiencias en materia de persecución efectiva de los denominados delitos de odio. En efecto, como ha denunciado la European Union Agency for Fundamental Rights, España se caracteriza por una acusada falta de atención a un fenómeno tan preocupante como ese, de lo que es muestra la propia carencia de estudios oficiales sobre su magnitud. No en vano, parece evidente que las normas penales introducidas para prevenir dicho fenómeno, como la agravante del art. 22.4 $4^{a}$ CP, o los tipos de los arts. 510-512 CP, se han mostrado incapaces de hacer frente a ese género de delincuencia. ${ }^{41}$

Se trata de dos retos urgentes, pero sin duda no son los únicos. Con todo, son dos cuestiones nucleares que, junto al análisis del carácter discriminatorio del funcionamiento de la punición, muestran un modo diferente de considerar la relación entre la diversidad cultural, el género y el sistema penal. Una forma alternativa, y probablemente de mayor utilidad social, que los parámetros habituales de debate, enmarcados en la retórica de la teoría de la delincuencia culturalmente motivada.

\footnotetext{
38 BRANDARIZ GARCÍA (2011), pp. 110 y ss.

39 Op. cit., p. 121 y s.

${ }^{40}$ FARRANT (2009), p. 124.

${ }^{41}$ BRANDARIZ GARCía (2010), pp. 48 y ss.
} 


\section{BIBLIOGRAFÍA}

* BAlibAR, Etienne y WALLERSTEIN, Immanuel (1991): Raza, nación y clase (Madrid, IEPALA).

* BAUMAN, Zygmunt (2010): Mundo consumo (Buenos Aires, Paidós).

* BRAH, Avtar (2011): Cartografías de la diáspora. (Madrid, Traficantes de Sueños).

* BRANDARIZ GARCÍA, José Ángel (2010): "Victimización de migrantes", en Josep Tamarit Sumalla (coord.), Victimas olvidadas (Valencia, Tirant lo Blanch) pp. 45-69.

* BRANDARIZ GARCÍA, José Ángel (2011): Sistema penal y control de los migrantes. Gramática del migrante como infractor penal (Granada, Comares).

* BRION, Fabienne (2010): “¿Utilizar el género para hacer la diferencia? La doctrina de los delitos culturales y de la defensa cultural”, en Salvatore Palidda y José Ángel Brandariz García (dirs.); Agustina Iglesias Skulj y José Antonio Ramos Vázquez (coords.): Criminalización racista de los migrantes en Europa (Granada, Comares) pp. 79-93.

* CARNEVALI, Raúl (2007): "El multiculturalismo: un desafío para el Derecho penal moderno", en Política Criminal (núm. 3) pp.1-28.

* CORNACCHIA, Luigi y SÁNCHEZ-OSTIZ, Pablo (coords.) (2012): Multiculturalismo y Derecho penal (Cizur Menor, Thomson Reuters Aranzadi)

* FARrANT, Finola (2009): “Gender, 'Race', and the Criminal Justice Process", en Hindpal Singh Bhui (ed.), Race and Criminal Justice (London, Sage) pp. 122-135.

* FEKETE, Liz (2009): A Suitable Enemy. Racism, Migration and Islamophobia en Europe (London/New York, Pluto Press).

* GOODEY, Jo (2006): "Ethnic profiling, criminal (in) justice and minority populations", en Critical Criminology (núm.14) pp. 207-2012.

* DE HAAN, Willem y VOS, Jaco (2003): “A crying shame: The over-rationalized conception of man in the rational choice perspective", en Theoretical Criminology (vol. 7, núm. 1) pp. 29-54.

* HOOKS, Bell et al. (2004): Otras inapropiables. Feminismos desde las fronteras (Madrid, Traficantes de Sueños).

* HUNTINGTON, Samuel (1996): The Clash of Civilizations and the Remaking of World Order (New York, Simon\&Schuster).

* IGLESIAS SKULJ, Agustina (2012): "Políticas públicas de lucha contra la trata de mujeres en el contexto español: luces y sombras", en Agustina Iglesias Skulj y Luz María Puente Aba (coords.), Sistema penal y perspectiva de género: trabajo sexual y trata de personas (Granada, Comares) pp. 61-80.

* JABARDO, Mercedes (ed.) (2012): Feminismos negros. Una antología (Madrid, Traficantes de Sueños).

* LAPARRA, Miguel (coord.) et al. (2011): Diagnóstico social de la comunidad gitana en España. (Madrid, Ministerio de Sanidad, Política Social e Igualdad) (disponible en: http:// wnw.msps.es/politicaSocial/inclusionSocial/poblacionGitana/docs/diagnosticosocial_autores.pdf).

* MOHANTY, Chandra (2008): "Bajo los ojos de occidente. Saber académico y discursos coloniales", en Sandro Mezzadra (coord.), Estudios postcoloniales. Ensayos fundamentales (Madrid, Traficantes de Sueños) pp. 69-101.

* MONCLÚS MASÓ, Marta (2008): La gestión penal de la inmigración (Buenos Aires, Del Puerto).

* NEXTGEnderation (2004): "Manifiesto NO EN NUESTRO NOMBRE" (disponible en: http:// www.nextgenderation.net/projects/notinournames/espanol.html).

* ObServatorio Metropolitano (2012): Spanish Neocon (Madrid, Traficantes de Sueños).

* PALIDDA, Salvatore (2008): Mobilitàumane (Milano, Raffaelo Cortina). 
Brandariz - Notas sobre la interseccionalidad entre etnicidad y políticas penales de género

* PÉREZ DEL VALLE, Carlos (2012): "Límites a la autonomía personal: ¿Existen bienes jurídicos indisponibles?”, en Luigi Cornacchia y Pablo Sánchez-Ostiz (coords.), Multiculturalismo y Derecho penal (Cizur Menor, Thomson Reuters Aranzadi) pp. 95-116.

* SCHMITT, Carl (2009): Teología politica (Madrid, Trotta).

* STOLCKE, Verena (1995): "Talking culture: new boundaries, new rhetorics of exclusion in Europe", en Current Anthropology (vol. 36, núm. 1) pp. 1-24.

* TSOUKALA, Anastassia (2002): "Le traitementmédiatique de la criminalitéétrangère en Europe”, en Déviance et Société (vol. 26, núm. 1) pp. 61-82.

* VÁZQUEZ GONZÁLEZ, Carlos (2010): Inmigración, diversidad y conflicto cultural (Madrid, Dykinson).

* YOUNG, Jock (2003): La sociedad "excluyente" (Madrid, Marcial Pons).

* YOUNG, Jock (2007): The Vertigo of Late Modernity (London, Sage).

* ŽIŽEK, Slavoj (2005): Bienvenidos al desierto de lo real (Madrid, Akal). 